\title{
Universiteit
}

Leiden

The Netherlands

\section{Decay of the Loschmidt Echo for quantum states with sub-Planck} scale structures

Beenakker, C.W.J.; Jacquod, Ph.; Adagideli, I.

\section{Citation}

Beenakker, C. W. J., Jacquod, P., \& Adagideli, I. (2002). Decay of the Loschmidt Echo for quantum states with sub-Planck scale structures. Retrieved from https://hdl.handle.net/1887/1272

Version: $\quad$ Not Applicable (or Unknown)

License: $\quad$ Leiden University Non-exclusive license

Downloaded from: https://hdl.handle.net/1887/1272

Note: To cite this publication please use the final published version (if applicable). 


\title{
Decay of the Loschmidt Echo for Quantum States with Sub-Planck-Scale Structures
}

\author{
Ph Jacquod, I Adagıdelı, and C W J Beenakke1 \\ Instituut-Lorentz Untversiteit Leiden, PO Box 9506, 2300 RA Leiden, The Netherlands
}

(Received 26 March 2002, published 20 September 2002)

\begin{abstract}
Quantum states extended over a large volume in phase space have oscrllations from quantum inteiferences in their Wigner distribution on scales smaller than $\hbar$ [W H Zurek, Nature (London) 412, 712 (2001)] We investigate the influence of those sub-Planck-scale structures on the sensitivity to an external peiturbation of the state's time evolution While we do find an accelerated decay of the Loschmidt Echo for an extended state in compaison to a localized wave packet, the acceleration is described entirely by the classical Lyapunov exponent and hence cannot originate from quantum interference
\end{abstract}

DOI $101103 /$ PhysRevLett 89154103

One common interpretation of the Heisenberg unceltainty principle is that phase-space stıuctures on scales smaller than $\hbar$ have no observable consequence The recent asser tion of Zuiek [1] that sub-Planck-scale stı uctures in the Wigner function enhance the sensitivity of a quantum state to an external perturbation, therefore, came out as particularly intinguing [2] and even controversial [3] His aigument can be summarized as follows The overlap (squared amplitude of the scalar product) of two quantum states $\psi$ and $\psi^{\prime}$ is given by the phase-space integial of the product of their Wignei functions,

$$
I_{\psi \psi^{\prime}} \equiv\left|\left\langle\psi \mid \psi^{\prime}\right\rangle\right|^{2}=(2 \pi \hbar)^{d} \int d \mathbf{r} d \mathbf{p} W_{\psi} W_{\psi^{\prime}}
$$

Fol an extended quantum state cover ing a large volume $A \gg \hbar^{d}$ of $2 d$-dimensional phase space, the Wigner function $W_{\psi}$ exhibits oscillations fiom quantum interferences on a scale corresponding to an action $\delta S \simeq \hbar^{2} / A^{1 / d} \ll \hbar$ These sub-Planck-scale oscillations are brought out of phase by a shift $\delta p, \delta x$ with $\delta p \delta x \simeq \delta S \ll \hbar$ The shifted state $\psi^{\prime}$ is then nearly orthogonal to $\psi$ since $I_{\psi \psi^{\prime}} \approx 0$ Zurek concludes that sub-Planck stiuctures substantially enhance the sensitivity of a quantum state to an external peiturbation

A measure of this sensitivity is piovided by the Loschmidt Echo $[4,5]$

$$
M(t)=\left|\left\langle\psi\left|\exp (\imath H t) \exp \left(-\imath H_{0} t\right)\right| \psi\right\rangle\right|^{2},
$$

giving the decaying overlap of two wave functions that stait out identically and evolve under the action of two slightly different Hamiltonians $H_{0}$ and $H=H_{0}+H_{1}$ (We set $\hbar=1$ ) One can interpiet $M(t)$ as the fidelity with which a quantum state can be reconstiucted by inverting the dynamics with a perturbed Hamiltonian In the context of envilonment-induced dephasing, $M(t)$ measures the decay of quantum inter ferences in a system with few degrees of fieedom interacting with an envilonment (with many more degiees of freedom) [6] In this case $\psi$ represents the state of the environment, which in
PACS numbers $0545 \mathrm{Mt}, 0545 \mathrm{Pq}, 0365 \mathrm{Yz}, 7660 \mathrm{Lz}$

general extends over a large volume of phase space This motıvated Karkuszewskı, Jarzynskı, and Zuıek [7] to investigate the dependence of $M(t)$ on short-scale structures

In this paper we study the same problem as in Ref [7], but ai1ive at opposite conclusions Finer and finei structures naturally develop in phase space when an initrally nat ow wave packet $\psi_{0}$ evolves in time under the influence of a chaotic Hamiltonian $H_{0}$ [7,8] As in Ref [7], we obset ve numeically a moie rapid decay of $M(t)$ for $\psi=$ $\exp \left(-\imath H_{0} T\right) \psi_{0}$ as the preparation time $T$ is made larger and laiger, with a satuiation at the Ehienfest time However, we demonstrate that this enhanced decay is described entriely by the classical Lyapunov exponent and hence is insensitive to the quantum interference that leads to the sub-Planck-scale structures in the Wigner function

In the case of a narrow initial wave packet, $M(t)$ has been calculated semiclassically by Jalabeit and Pastawskı [5] Before discussing extended states with short-scale stiuctures, we recapitulate their calculation The time evolution of a wave packet centered at $\mathbf{r}_{0}$ is approximated by

$$
\begin{gathered}
\psi(\mathbf{r}, t)=\int d \mathbf{r}_{0} \sum_{s} K_{s}^{H}\left(\mathbf{r}, \mathbf{r}_{0}, t\right) \psi_{0}\left(\mathbf{r}_{0}\right), \\
K_{s}^{H}\left(\mathbf{r}, \mathbf{r}_{0}, t\right)=C_{s}^{1 / 2} \exp \left[\imath S_{s}^{H}\left(\mathbf{r}, \mathbf{r}_{0}, t\right)-\imath \pi \mu_{s} / 2\right]
\end{gathered}
$$

The semiclassical propagator is a sum over classical trajectories (labeled $s$ ) that connect $\mathbf{r}$ and $\mathbf{r}_{0}$ in the time $t$ For each $s$, the partial piopagator is expiessed in teims of the action integial $S_{s}^{H}\left(\mathbf{r}, \mathbf{r}_{0}, t\right)$ along $s$, a Maslov index $\mu_{s}$ (which will diop out), and the determinant $C_{s}$ of the monodromy mat11x After a stationary phase appioximation, one gets

$$
M(t) \simeq\left|\int d \mathbf{r} \sum_{s} K_{s}^{H}\left(\mathbf{r}, \mathbf{r}_{0}, t\right)^{\times} K_{s}^{H_{0}}\left(\mathbf{r}, \mathbf{r}_{0}, t\right)\right|^{2}
$$

Squaing the amplitude in Eq (5) leads to a double sum 
over classical paths $s, s^{\prime}$ and a double integration over final coordinates $\mathbf{r}, \mathbf{r}^{\prime}$. Accordingly, $M(t)$ splits into diagonal $\left(s=s^{\prime}, \mathbf{r}=\mathbf{r}^{\prime}\right)$ and nondiagonal $\left(s \neq s^{\prime}\right.$ or $\left.\mathbf{r} \neq \mathbf{r}^{\prime}\right)$ contributions. Since quantum phases entirely drop out of the diagonal contribution, its decay is solely determined by the classical quantity $C_{s} \propto \exp (-\lambda t)$. Here $\lambda$ is the Lyapunov exponent of the classical chaotic dynamics, which we assume is the same for $H$ and $H_{0}$. The nondiagonal contribution also leads to an exponential decay, which however originates from the phase difference accumulated when traveling along a classical path with two different Hamiltonians [5]. The slope $\Gamma$ of this decay is the golden rule spreading the width of an eigenstate of $H_{0}$ over the eigenbasis of $H[9,10]$. Since $M(t)$ is given by the sum of these two exponentials, the Lyapunov decay will prevail for $\Gamma>\lambda$.

The Lyapunov decay sensitively depends on the choice of an initial narrow wave packet $\psi_{0}$ [11]. The faster decay of $M(t)$ resulting from the increased complexity of the initial state can be quantitatively investigated by considering prepared states $\psi=\exp \left(-i H_{0} T\right) \psi_{0}$, i.e., narrow wave packets that propagate during a time $T$ with the Hamiltonian $H_{0}[12]$, thereby developing finer and finer structures in phase space as $T$ increases $[7,8]$. The stationary phase approximation to the fidelity then reads

$$
M_{T}(t)=\left|\int d \mathbf{r} \sum_{s} K_{s}^{H_{\tau}}\left(\mathbf{r}, \mathbf{r}_{0} ; t+T\right)^{\succ} K_{s}^{H_{0}}\left(\mathbf{r}, \mathbf{r}_{0} ; t+T\right)\right|^{2}
$$

with the time-dependent Hamiltonian $H_{\tau}=H_{0}$ for $\tau<T$ and $H_{\tau}=H$ for $\tau>T$.

We can apply the same analysis as in Ref. [5] to the time-dependent Hamiltonian. Only the time interval $(T, t+T)$ of length $t$ leads to a phase difference between $K_{s}^{H_{\tau}}$ and $K_{s}^{H_{0}}$, because $H_{\tau}=H_{0}$ for $\tau<T$. Hence the nondiagonal contribution to $M_{T}(t)$, which is entirely due to this phase difference, still decays $\propto \exp (-\Gamma t)$, independent of the preparation time $T$. We will see below that this is in agreement with a fully quantum mechanical approach according to which the golden rule decay is independent of the complexity of the initial state.

The preparation does however have an effect on the diagonal contribution $M_{T}^{(d)}(t)$ to the fidelity. It decays $\propto$ $\exp [-\lambda(t+T)]$ instead of $\propto \exp (-\lambda t)$, provided $t, T \gg$ $\lambda^{-1}$. This is most easily seen from the expression

$$
M_{T}^{(d)}(t)=\int d \mathbf{r} \sum_{s}\left|K_{s}^{H_{\tau}}\left(\mathbf{r}, \mathbf{r}_{0} ; t+T\right)\right|^{2}\left|K_{s}^{H_{0}}\left(\mathbf{r}, \mathbf{r}_{0} ; t+T\right)\right|^{2},
$$

by following a path from its endpoint $\mathbf{r}$ to an intermediate point $\mathbf{r}_{\imath}$ reached after a time $t$. The time evolution from $\mathbf{r}$ to $\mathbf{r}_{i}$ leads to an exponential decrease $\propto \exp (-\lambda t)$ as in Ref. [5]. Because of the classical chaoticity of $H_{0}$, the subsequent evolution from $\mathbf{r}_{1}$ to $\mathbf{r}_{0}$ in a time $T$ brings in an additional prefactor $\exp (-\lambda T)$.

The combination of diagonal and nondiagonal contributions results in the biexponential decay (valid for $\Gamma t, \lambda t$, $\lambda T \gg 1)$

$$
M_{T}(t)=A(t) \exp (-\Gamma t)+B(t) \exp [-\lambda(t+T)],
$$

with prefactors $A$ and $B$ that depend algebraically on time. The Lyapunov decay prevails if $\Gamma>\lambda$ and $t>\lambda T /(\Gamma-$ $\lambda)$, while the golden rule decay dominates if either $\Gamma<\lambda$ or $t<\lambda T /(\Gamma-\lambda)$. In both regimes the decay saturates when $M_{T}$ has reached its minimal value $1 / I$, where $I$ is the total accessible volume of phase space in units of $\hbar^{d}$. In the Lyapunov regime, this saturation occurs at $t=$ $t_{E}-T$, where $t_{E}=\lambda^{-1} \ln I$ is the Ehrenfest time. When the preparation time $T \rightarrow t_{E}$, we have a complete decay within a time $\lambda^{-1}$ of the fidelity down to its minimal value.

We now present numerical checks of these analytical results for the Hamiltonian

$$
H_{0}=\left(\pi / 2 \tau_{0}\right) S_{y}+(K / 2 S) S_{z}^{2} \sum_{n} \delta\left(t-n \tau_{0}\right) .
$$

This kicked top model [13] describes a vector spin of magnitude $S$ undergoing a free precession around the $y$ axis and being periodically perturbed (period $\tau_{0}$ ) by sudden rotations around the $z$ axis over an angle proportional to $S_{z}$. The time evolution after $n$ periods is given by the $n$th power of the Floquet operator

$$
F_{0}=\exp \left[-i(K / 2 S) S_{z}^{2}\right] \exp \left[-i(\pi / 2) S_{y}\right]
$$

Depending on the kicking strength $K$, the classical dynamics is regular, partially chaotic, or fully chaotic. We perturb the reversed time evolution by a periodic rotation of constant angle around the $x$ axis, slightly delayed with respect to the kicks in $H_{0}$,

$$
H_{1}=\phi S_{x} \sum_{n} \delta\left(t-n \tau_{0}-\epsilon\right)
$$

The corresponding Floquet operator is $F=$ $\exp \left(-i \phi S_{\lambda}\right) F_{0}$. We set $\tau_{0}=1$ for ease of notation. We took $S=500$ (both $H$ and $H_{0}$ conserve the spin magnitude, the corresponding phase space being the sphere of radius $S$ ) and calculated the averaged decay $\bar{M}_{T}$ of $M_{T}(t=n)=\left|\left\langle\psi\left|\left(F^{\dagger}\right)^{n} F_{0}^{n}\right| \psi\right\rangle\right|^{2}$ taken over 100 initial states.

We choose $\psi_{0}$ as a Gaussian wave packet (coherent state) centered on a point $(\theta, \varphi)$ in spherical coordinates. The state is then prepared as $\psi=\exp \left(-i H_{0} T\right) \psi_{0}$. We can reach the Lyapunov regime by selecting initial wave packets centered in the chaotic region of the mixed phase space for the Hamiltonian (9) with kicking strength $K=$ 3.9 [9]. Figure 1 gives a clear confirmation of the predicted decay $\propto \exp [-\lambda(t+T)]$ in the Lyapunov regime. The additional decay induced by the preparation time $T$ 


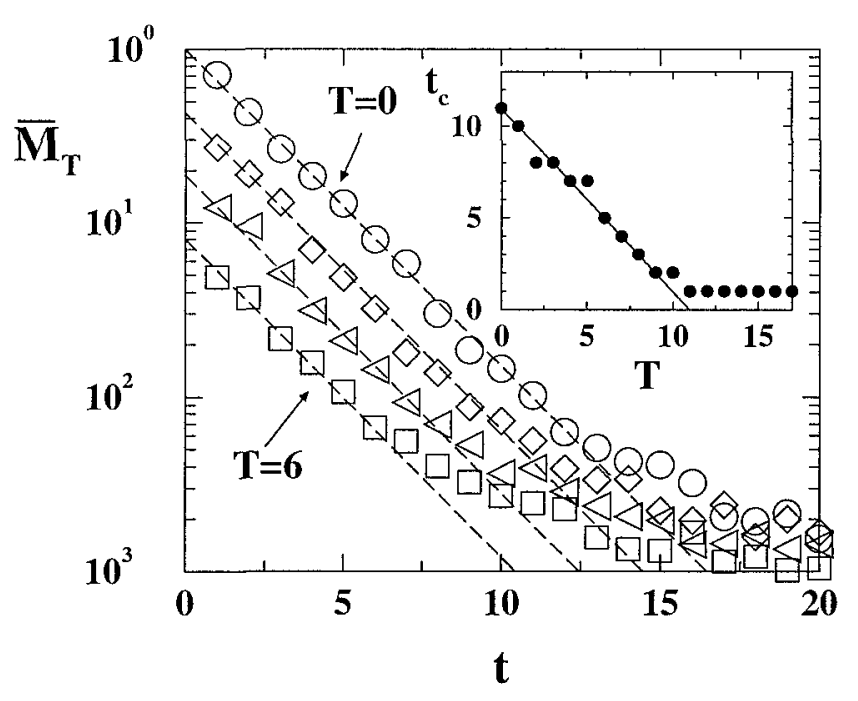

FIG 1 Decay of the average fidelity $\bar{M}_{T}$ for the kicked top with parameters $\phi=12 \times 10^{-3}, K=39$ and for preparation tumes $T=0$ (circles), 2 (diamonds), 4 (triangles), and 6 (squales) In each case, the dashed lines give the analytical decay $\vec{M}_{T}=\exp [-\lambda(t+T)]$, in the Lyapunov legime with $\lambda=042$ Inset threshold time at which $\bar{M}_{T}\left(t_{c}\right)=M_{c}=$ $10^{-2}$ The solid line gives the analytical behavior $t_{c}=$ $-\lambda{ }^{1} \ln M_{c}-T$

can be quantified via the time $t_{c}$ it takes for $\bar{M}_{T}$ to reach a given thieshold $M_{c}$ [7] We expect

$$
t_{c}=-\lambda^{-1} \ln M_{c}-T
$$

provided $M_{c}>1 / I=1 / 2 S$ and $T<-\lambda^{-1} \ln M_{c}$ In the inset of $F_{1} g 1$ we confirm this formula for $M_{c}=10^{-2}$ As expected, $t_{c}$ satuiates at the first $\operatorname{kick}\left(t_{c}=1\right)$ when $T \simeq$ $-\lambda^{-1} \ln M_{c}<t_{E}$ Numerical results qualitatively similar to those shown in the inset to Fig 1 [14] were obtained in Ref [7] and interpieted there as the accelerated decay 1esulting fiom sub-Planck-scale structures The fact that our numerical data are described so well by Eq (12) points to a classical 1 ather than a quantum oilgin of the decay acceleiation Indeed, $\mathrm{Eq} \mathrm{(12)} \mathrm{contains} \mathrm{only} \mathrm{the}$ classical Lyapunov exponent as a system dependent parametel, so that it cannot be sensitive to any fine sti ucture in phase space resulting fiom quantum inter ference

We next illustrate the independence of $M_{T}(t)$ on the preparation time $T$ in the golden rule iegime, $1 \mathrm{e}$, at laige k1cking stiength $K$ when $\lambda>\Gamma$ [9] As shown in Fig 2, the decay of $M_{T}(t)$ is the same for the four different preparation times $T=0,5,10$, and 20 We estimate the Ehienfest time as $t_{E} \approx 7$, so that increasing $T$ further does not inciease the complexity of the initial state

These numerical data give a clear confiımation of the semiclassical result (8) Previous investigations have established the existence of five different regimes for the decay of $M(t)[4,5,9,10,15]$, and since only two of them aie captured by the semiclassical approach used above, we now show that short-scale structures do not affect the lemaining three The five regimes corlespond to different decays (1) Parabolic decay, $M(t)=1-\sigma^{2} t^{2}$, with $\sigma^{2} \equiv$ $\left\langle\psi_{0}\left|H_{1}^{2}\right| \psi_{0}\right\rangle-\left\langle\psi_{0}\left|H_{1}\right| \psi_{0}\right\rangle^{2}$, which exists for any perturbation stiength at short enough times (11) Gaussian decay, $M(t) \propto \exp \left(-\sigma^{2} t^{2}\right)$, valid if $\sigma$ is much smalle than the level spacing $\Delta$ (111) Golden 1 ule decay, $M(t) \propto$ $\exp (-\Gamma t)$, with $\Gamma \simeq \sigma^{2} / \Delta$, if $\Delta<\Gamma<\lambda$ (iv) Lyapunov decay, $M(t) \propto \exp (-\lambda t)$, if $\lambda<\Gamma$ (v) Gaussian decay, $M(t) \propto \exp \left(-B^{2} t^{2}\right)$, if $H_{1}$ is so laige that $\Gamma$ is laigel than the energy bandwidth $B$ of $H$

All these regimes except regime (iv) can be dealt with quantum mechanically under the sole assumption that both $H_{0}$ and $H$ are classically chaotic, using 1andom matrix theory (RMT) [16] Both sets of eigenstates $|\alpha\rangle$ of $H$ (with $N$ eigenvalues $\epsilon_{\alpha}$ ) and $\left|\alpha_{0}\right\rangle$ of $H_{0}$ (with $N$ eigenvalues $\epsilon_{\alpha}^{0}$ ) are then iotationally invariant [17] Expanding $\psi=\sum_{\alpha} \psi_{\alpha}|\alpha\rangle$ and assuming unbioken time-1eveisal symmetry, the fidelity (2) can be iewritten as

$$
M(t)=\sum_{\alpha \beta \gamma \delta} \psi_{\alpha} \psi_{\beta} \psi_{\gamma} \psi_{\delta}\left\langle\alpha\left|\exp \left(-{ }_{\imath} H_{0} t\right)\right| \beta\right\rangle\left\langle\gamma\left|\exp \left(\iota H_{0} t\right)\right| \delta\right\rangle \exp \left[l\left(\epsilon_{\alpha}-\epsilon_{\delta}\right) t\right]
$$

RMT implies the $\psi$-independent average $\overline{\psi_{\alpha} \psi_{\beta} \psi_{\gamma} \psi_{\delta}}=$ $\left(\delta_{\alpha \beta} \delta_{\gamma \delta}+\delta_{\alpha \gamma} \delta_{\beta \delta}+\delta_{\alpha \delta} \delta_{\beta \gamma}\right) / N^{2}$ The thrid contiaction gives a contilbution $N^{-1}$ repiesenting the satuiation of $M(t)$ for $t \rightarrow \infty$ The other two give the time dependence

$$
\bar{M}(t)=N^{-1}+\left.\left.2 N^{-2}\left|\sum_{\alpha \beta_{0}}\right|\left\langle\alpha \mid \beta_{0}\right\rangle\right|^{2} \exp \left[t\left(\epsilon_{\alpha}-\epsilon_{\beta}^{0}\right) t\right]\right|^{2}
$$

For per tur batively weak $H_{1}$ one has $\epsilon_{\alpha}=\epsilon_{\alpha}^{0}+\left\langle\alpha\left|H_{1}\right| \alpha\right\rangle$ and $\left\langle\alpha \mid \beta_{0}\right\rangle=\delta_{\alpha \beta_{0}}$ According to RMT the matix elements $\left\langle\alpha\left|H_{1}\right| \alpha\right\rangle$ are independent random numbers, and for laige $N$ the central limit theorem leads to the Gaussian decay (11) [or the parabolic decay (1) for short times] At laiger perturbation stiength, $\left|\left\langle\alpha \mid \beta_{0}\right\rangle\right|^{2}$ becomes
Loientzian,

$$
\left|\left\langle\alpha \mid \beta_{0}\right\rangle\right|^{2}=\frac{\Gamma / 2 \pi}{\left(\epsilon_{\alpha}-\epsilon_{\beta}^{0}\right)^{2}+\Gamma^{2} / 4},
$$

with a width $\Gamma \simeq \overline{\left.\left\langle\alpha_{0}\left|H_{1}\right| \beta\right\rangle\right|^{2}} / \Delta$ given by the golden 1 ule This leads to regime (111) Increasing $H_{1}$ further, one obtains an ergodic distiıbution $\overline{\left|\left\langle\alpha \mid \beta_{0}\right\rangle\right|^{2}}=N^{-1}$ and a straightfor waid calculation produces regime (v) This establishes that, under the sole assumption that $H_{0}$ and $H$ aie classically chaotic, the decay of the fidelity in the thiee quantum regimes (11), (111), and (v) does not depend on the choice of the initial state $\psi$ 


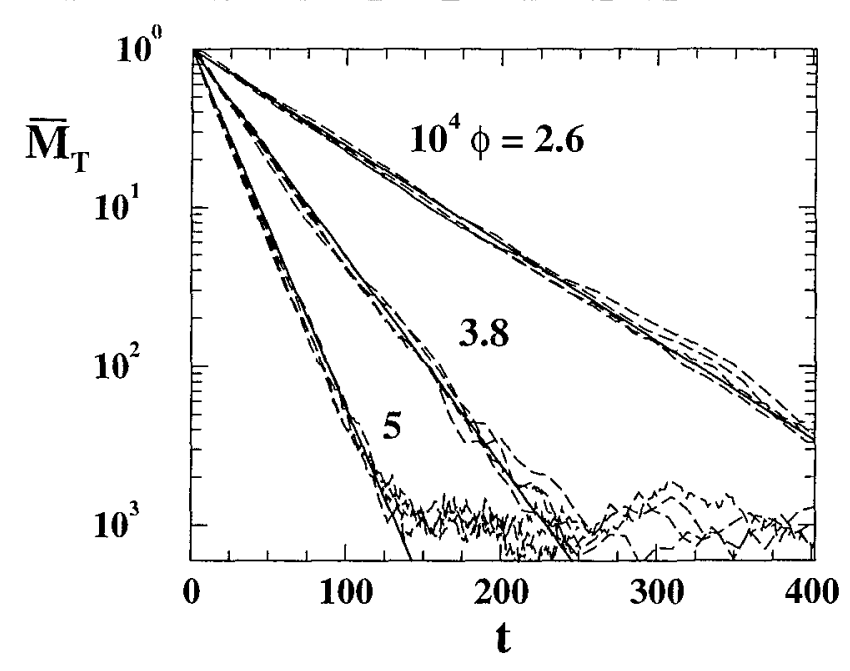

FIG. 2. Decay of $\bar{M}_{T}$ in the golden rule regime for $\phi=2.6 \times$ $10^{-4}, 3.8 \times 10^{-4}, 5 \times 10^{-4}, K=13.1$, and for preparation times $T=0,5,10$, and 20 (nearly indistinguishable dashed lines). The solid lines give the corresponding golden rule decay with $\Gamma=0.84 \phi^{2} S^{2}[9]$.

In summary, we have investigated the decay of the Loschmidt Echo, Eq. (2), for quantum states $\psi=$ $\exp \left(-i H_{0} T\right) \psi_{0}$ that have spread over phase space for a time $T$. As in Ref. [7], we found a faster decay of $M_{T}(t)$ than for a localized wave packet, but only in the regime where the decay rate is set by the classical Lyapunov exponent $\lambda$. Since quantum interferences play no role in this regime, we conclude that sub-Planck-scale structures in the Wigner representation of $\psi$ do not influence the decay of the Loschmidt Echo.

This work was supported by the Swiss National Science Foundation, the Dutch Science Foundation NWO/FOM, and by the U.S. Army Research Office. We acknowledge helpful discussions with N. Cerruti, R. A. Jalabert, and S. Tomsovic.

[1] W. H. Zurek, Nature (London) 412, 712 (2001).

[2] A. Albrecht, Nature (London) 412, 687 (2001).
[3] A. Jordan and M. Srednicki, quant-ph/0112139.

[4] A. Peres, Phys. Rev. A 30, 1610 (1984).

[5] R. A. Jalabert and H. M. Pastawski, Phys. Rev. Lett. 86, 2490 (2001).

[6] E. Joos and H. D. Zeh, Z. Phys. B 59, 223 (1985).

[7] Z.P. Karkuszewski, C. Jarzynski, and W. H. Zurek, quant-ph/0111002.

[8] G. P. Berman and G. M. Zaslavsky, Physica (Amsterdam) 91A, 450 (1978); M. V. Berry and N. L. Balasz, J. Phys. A 12, 625 (1979).

[9] Ph. Jacquod, P. G. Silvestrov, and C.W. J. Beenakker, Phys. Rev. E 64, 055203(R) (2001).

[10] F. M. Cucchietti, C. H. Lewenkopf, E. R. Mucciolo, H. M. Pastawski, and R. O. Vallejos, Phys. Rev. E 65, 046209 (2002).

[11] For example, if $\psi_{0}$ is a coherent superposition of $N$ wave packets, the diagonal (Lyapunov) contribution is reduced by a factor $1 / N$ while the off-diagonal (golden rule) contribution remains the same.

[12] More generally, we could prepare the state $\psi=$ $\exp \left(-i H_{p} T\right) \psi_{0}$ with a chaotic Hamiltonian $H_{p}$ that is different from $H_{0}$ and $H$. We assume $H_{p}=H_{0}$ for ease of notation, but our results are straightforwardly extended to this more general case.

[13] F. Haake, Quantum Signatures of Chaos (Springer, Berlin, 2000).

[14] The similarity between the data in our Fig. 1 and in Ref. [7] is only qualitative, mainly because of the much larger value $M_{c}=0.9$ chosen in Ref. [7]. For values of $M_{c}$ close to 1 , we expect that we can do perturbation theory in $t$ which gives $M_{T}(t)=1-\exp (\lambda T) \sigma^{2} t^{2}$, and hence $t_{c}=\sqrt{1-M_{c}} \exp (-\lambda T / 2) / \sigma$. Analyzing the data presented in Fig. 2 of Ref. [7] gives the values $\sigma \approx 0.042$ and $\lambda \approx 0.247$.

[15] N. Cerruti and S. Tomsovic, Phys. Rev. Lett. 88, 054103 (2002); T. Prosen and M. Znidaric, J. Phys. A 35, 1455 (2002); T. Prosen and T. Seligman, nlin.CD/0201038; G. Benenti and G. Casati, Phys. Rev. E 65, 066205 (2002); D. A. Wisniacki and D. Cohen, nlin.CD/0111125.

[16] The RMT assumption relates the fidelity to the local spectral density of states. The Lyapunov regime is however not captured by this relationship; see D. Cohen, Phys. Rev. E 65, 026218 (2001).

[17] M. L. Mehta, Random Matrices (Academic, New York, 1991). 